JAIST Repository

https://dspace.jaist.ac.jp/

\begin{tabular}{|c|c|}
\hline Title & $\begin{array}{l}\text { Augment ed cl assroom A paper-centric approach for } \\
\text { col I abor at i ve I earni ng syst em }\end{array}$ \\
\hline Author(s) & $\begin{array}{l}\text { Mura, M. Kuni fuj i, Susumi; Shi zuki, B.; Tanaka, } \\
\text { J. }\end{array}$ \\
\hline Citation & Lect ure Not es in Comput er Sci ence, 3598: 104116 \\
\hline Issue Date & 2005 \\
\hline Type & Journal Arti cle \\
\hline Text version & aut hor \\
\hline URL & ht t p: //hdl . handl e. net /10119/3422 \\
\hline Rights & $\begin{array}{l}\text { Thi s i s the aut hor-creat ed ver si on of Spr i nger } \\
\text { Berl i n / Hei del ber g, Not oki M ur a, Susumi } \\
\text { Kuni f uj i , Bunt ar ou Shi zuki and Ji ro Tanaka, } \\
\text { Lect ur e Not es i n Comput er Sci ence( Ubi qui t ous } \\
\text { Comput i ng Syst ens), 3598, 2005, 104 116. The } \\
\text { or i gi nal publ i cat i on is avai I abl e at } \\
\text { www. spr i nger I i nk. com } \\
\text { ht t ps: //uww. spr i nger l i nk. com/cont ent /df axx8l au625 } \\
\text { wr 78/r esour ce- secur ed/?t ar get =f ul I t ext. pdf }\end{array}$ \\
\hline Des & \\
\hline
\end{tabular}

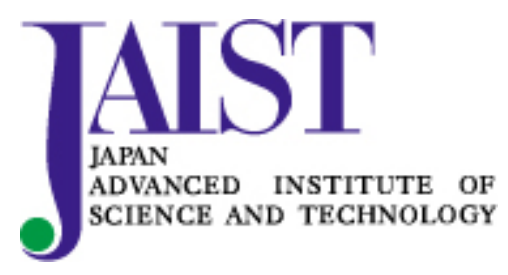




\title{
Augmented Classroom: A Paper-Centric Approach for Collaborative Learning System
}

\author{
Motoki Miura Susumu Kunifuji \\ School of Knowledge Science \\ Japan Advanced Institute of Science and Technology \\ 1-1 Asahidai, Tatsunokuchi, Nomi, Ishikawa, 923-1292, Japan \\ \{miuramo, kuni\}@jaist.ac.jp \\ Buntarou Shizuki Jiro Tanaka \\ Department of Computer Science \\ Graduate School of Systems and Information Engineering \\ University of Tsukuba \\ 1-1-1 Tennodai, Tsukuba, Ibaraki, 305-8573, Japan \\ \{shizuki,jiro\}@cs.tsukuba.ac.jp
}

\begin{abstract}
We developed AirTransNote, a computer-mediated classroom collaboration system. The system enables real-time note-sharing. AirTransNote manages notes written by students on paper and enables the teacher to browse through the notes or show them to the students. AirTransNote can analyze students' answers, helping the teacher better understand their problems. The system is not meant to provide an alternative to the conventional way of instruction; rather, it is designed to enhance class interaction. We conducted a preliminary study using questionnaires and found that this system can be feasible to apply for classroom environment.
\end{abstract}

\section{Introduction}

In the last decades, many educational institutions have started to introduce computers into the classroom. Most schools in Japan already have PC rooms for computer literacy classes, collaborative activities, and individual study. However, computers have not yet become part of everyday school life because they can seldom be used outside the $\mathrm{PC}$ room.

There is an increasing interest among school professionals and administrators in using computers for everyday classes. For example, The Computers in Education project in Japan [1] is designed to promote the use of network-connected computers and LCD projectors in the regular classroom. Computers are used to show digitally the main points of lectures through images, short video clips, animation, and other applications. The use of computers for demonstration purposes is justified because it helps students better under- stand the content of lectures. Yet in this approach, the use of computers in the classroom is limited.

We believe that the main purpose of using computers is to promote interaction among students and teachers and create a collaborative learning environment. To enable student participation in computer-mediated learning, classrooms must be equipped with a sufficient number of computers. However, there is usually not enough room in a typical classroom to install computers for all students. Also, devices such as PCs and PDAs which require special skills to operate are not appropriate for students.

\section{System "AirTransNote"}

We developed a computer-mediated collaborative learning system called AirTransNote. AirTransNote is designed to augment the traditional way of communication in the classroom. Air- 


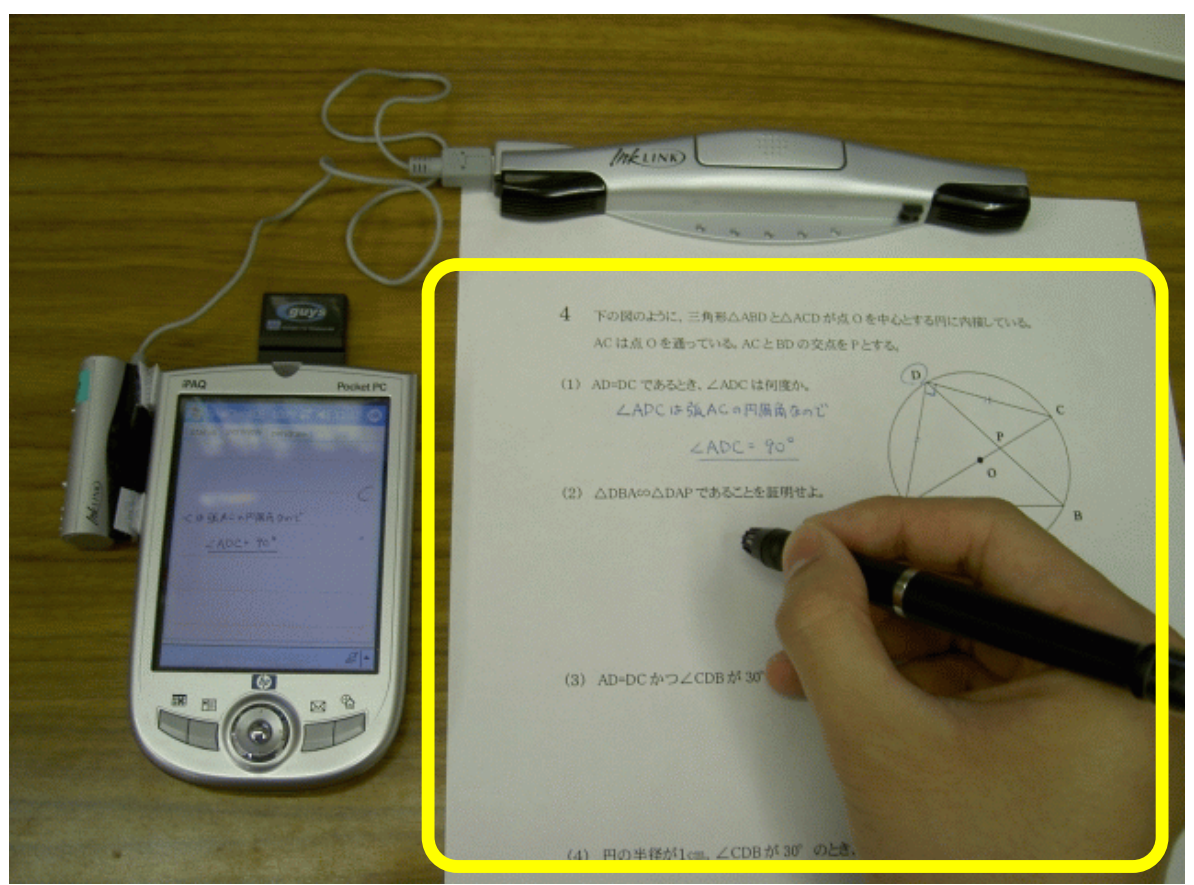

Figure 1: Transmitters in AirTransNote. The rectangle indicates the area on which the student should concentrate.

TransNote is similar to systems designed for chat-augmented conferences[2]. We believe that the system can be incorporated into conventional classrooms to become part of everyday class interaction and learning.

AirTransNote enables real-time sharing of notes. When a student writes notes on a regular sheet of paper, the handwritten data are immediately sent to the teacher's computer. In our current implementation, the transmitter in AirTransNote is a PDA with a wireless local area network connection and a digital pen. The digital pen is a device which is used to write notes and scribbles on paper. The pen enables recording the coordinates of notes and the timestamp in digital form. The notes are stored into the local memory of the PDA first, and then the notes are transferred to PC by an explicit operation for synchronization. AirTransNote stores data into the local memory of the PDA for backup purposes, but the data are sent to the server in real time. Successive transfer of data improves the effectiveness of communication. We used this feature for interactive communication among the teacher and students.

\subsection{Transmitter (client)}

Figure 1 shows the transmitter of AirTransNote. It combines a digital pen (InkLink[3]) and a PDA with a wireless LAN device. The digital pen consists of a sensor, an IrDA relay, and a regular pen.

The transmitter works as follows. Using a clip, a student attaches a sheet of paper to the sensor and connects the sensor to the IrDA relay. Then the student arranges the IrDA relay and PDA in such a way that an infrared connection is established.

Basically, the transmitter is designed to free the student from the need to operate the PDA during class. The only operation the student needs to perform is pressing a hot-key on PDA at the beginning of the class. The hot-key wakes up the PDA device and activates the AirTransNote transmitter.

When the student puts the pen down, ultrasonic waves are generated from the tip of the pen. The sensor recognizes the waves and calculates the current position of the pen tip. The tip position is transmitted to the PDA via the IrDA relay. Finally, the PDA transfers the data to the server through the wireless LAN device.

\subsection{Manager (server)}

Figure 2 shows the note-browsing interface of the AirTransNote manager. Transmitted notes are received by the manager, who immediately updates the browser by adding the notes to the corresponding student panel. The teacher can use the browser not only to check the students' progress, but also 


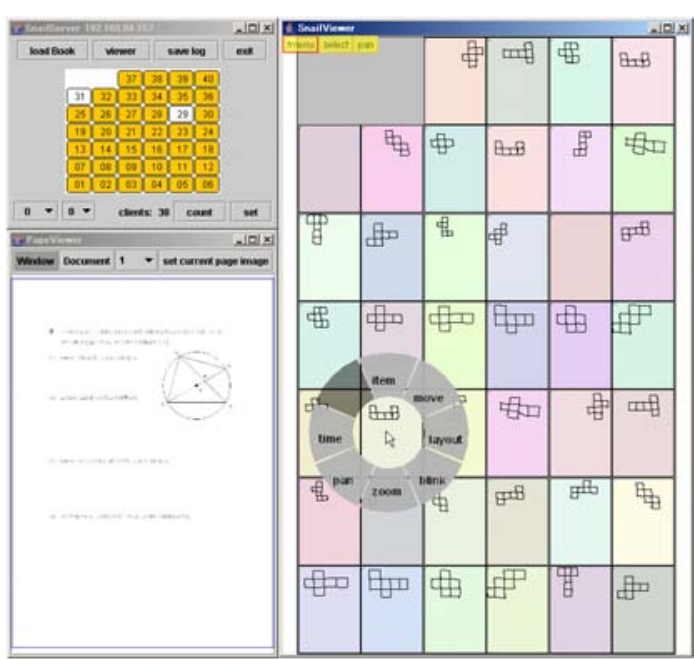

Figure 2: Note-browsing interface in the AirTransNote manager

to show them the notes with explanations.

The student panels have the form of a regular paper sheet. When the teacher activates a student panel by clicking on it, the browser zooms in onto the activated student panel. The zoom level and focus can be easily adjusted by using FlowMenu[4] and a mouse with a wheel. So that the class could better understand the notes, they can be displayed in an image of content printed on the answer sheet (see Figure 3). The teacher can choose the image and apply it using a page chooser (bottom left corner in Figure 2).

The upper left corner in Figure 2 shows the connection status of each client (a student number filled by yellow color means connected). The status window is also used for shortcuts to turn on an activated student panel by clicking on the corresponding student number. When the teacher uses this shortcut, the view in the current student panel is preserved in the new activated panel. The teacher can easily zoom in on part of the answer sheet in each student's panel by selecting a shortcut. Panning, zooming, and switching operations are performed with smooth animated transitions in the note browser.

In addition to the notes, a timestamp of stroke can also be used for a time-based presentation. AirTransNote can replay notes at different speeds, which enables time-based visualization of notes. The teacher can draw students' attention to a particular answer by using a time-line bar.

AirTransNote can be used to present ideas and solutions through note-sharing and it is particularly useful in the classroom. This system can be

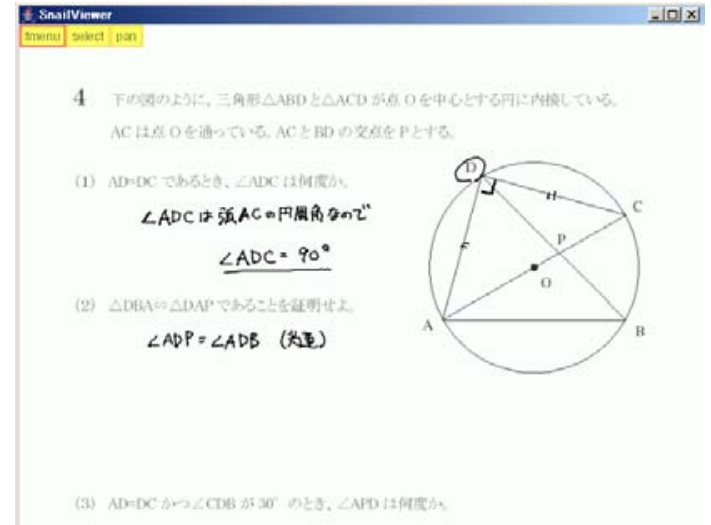

Figure 3: Zoomed-in view of notes overlapped with a printed-paper image

used, for example, in geometry class, helping the teacher evaluate students' drawings. The teacher can zoom in on a particular answer, explain solutions, and comment on student answers and drawings.

Although the interface of the manager is fairly sophisticated, there is still not enough time for the teacher to check the work of all students during a class period. We thus added an analysis function to AirTransNote. This function provides automatic recognition and presentation of results. We implemented the recognition module based on a simple region-matching algorithm; two visual presentation modules enable viewing the results in table or graph form. These modules can be used to collect results of a multiple-choice questionnaire or to check students' progress on paper tests. Figure 4 shows a graph view and table view of a result generated by the modules. Both the graph and table views are constantly updated to reflect the latest results in the recognition module. The table rows can be sorted by specifying the needed column. The teacher can transfer the data in the table into a cvs file.

The analysis function is useful because it helps the teacher quickly understand the progress of each student. Based on the responses, the teacher can control the speed of the lecture. Also the manager can issue commands to play sounds at the transmitter's side. This function can be used to inform students of test results as soon as they become available. 


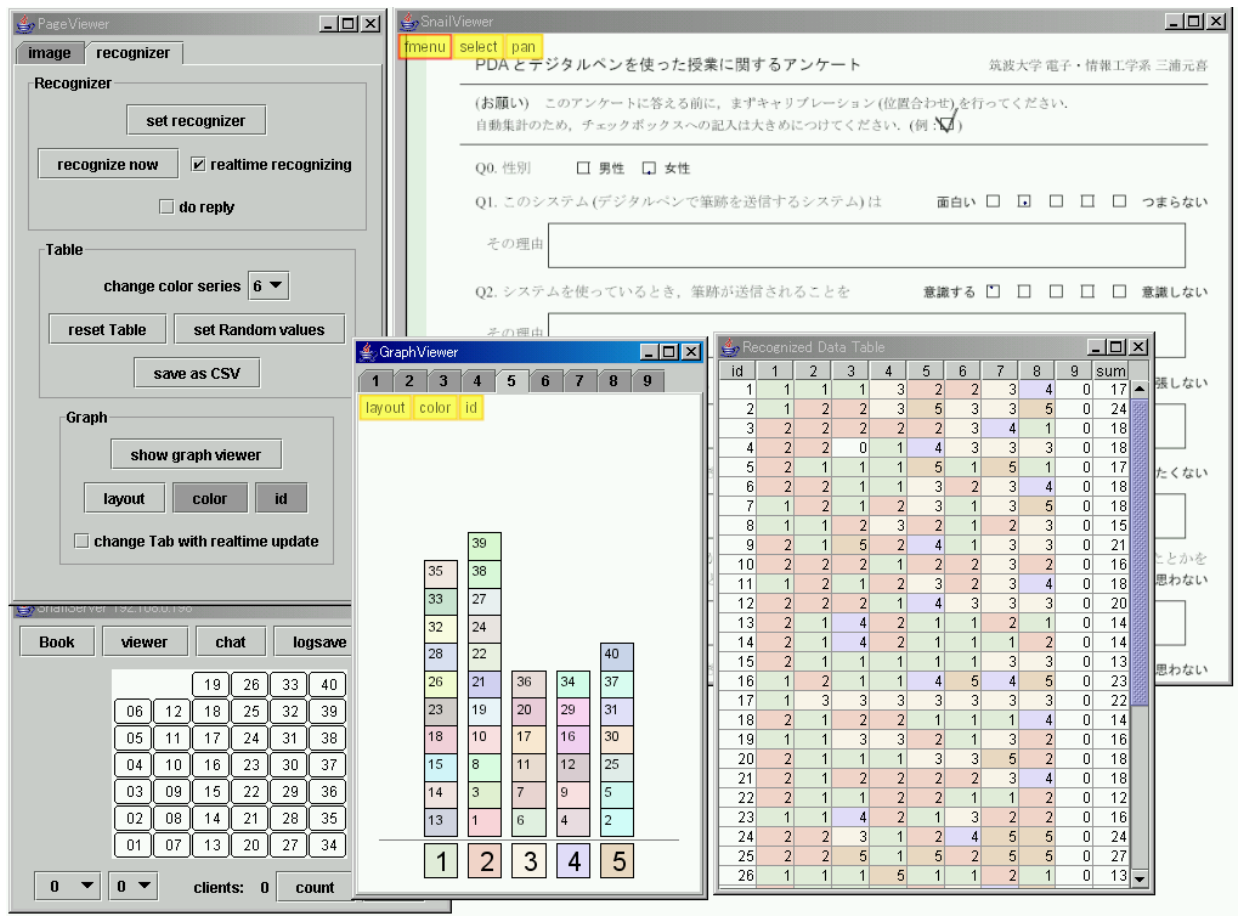

Figure 4: Summary of questionnaire results presented in graph and table forms.

\subsection{Advantages}

\subsubsection{Advantages to students}

The most useful feature of the paper-centric approach is that it frees the student from the need to use digital devices while preserving the traditional style of teaching with paper and pen. The goal of computer-mediated learning is to maximize the efficiency of the learning process and facilitate the acquisition of knowledge by using technology, not to make the student acclimate to the devices. Most conventional PC- or PDA-based learning technologies require that students use electronic devices. As a result, students who are not familiar with such devices may be at a disadvantage. Our system based on the paper-centric approach provides collaborative learning functions with a natural interface that is easy to use.

\subsubsection{Advantages to teachers}

We respect the traditional way of teaching and at the same time believe that it should be enhanced. Our system is designed to supplement the traditional teaching style with real-time collaboration. Teachers can use our system to supplement the more conventional instruction tools such as paperbased lecture materials.
In a classroom with many students, it may not be possible for the teacher to pay close attention to every student. Our system solves this problem by providing the teacher with a means to check students' work. By viewing test results presented in graph form, the teacher can see which students failed to solve which problems and can direct his/her attention to those students. A detailed log can be used to categorize test mistakes and problems so that the class could go over them in the future. This is also advantageous to students.

The system consists of a digital pen device with a PDA, a wave-LAN station, a projector, and a PC for the teacher. Assuming that both the digital pens and PDAs have been distributed among the students, and the wave-LAN station has been installed in the classroom, the system can be set up very quickly.

\subsection{Implementation}

\subsubsection{Transmitter (client)}

We developed the transmitter using Microsoft eMbedded Visual $\mathrm{C}++4.0$. The transmitter runs on a Pocket PC 2003 with an IrDA port. To collect notes created with a digital pen, we used InkNote Manager SDK made by Seiko Instruments USA 


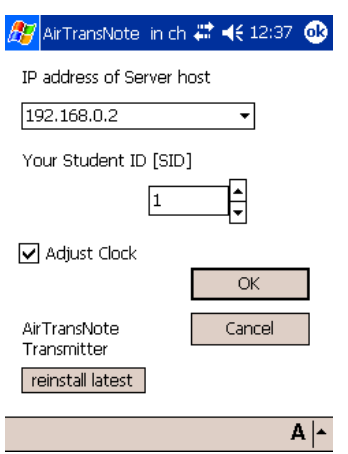

(a)

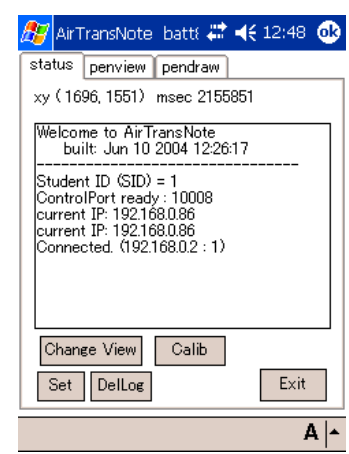

(b)

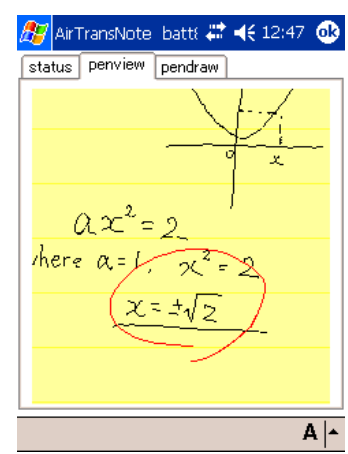

(c)

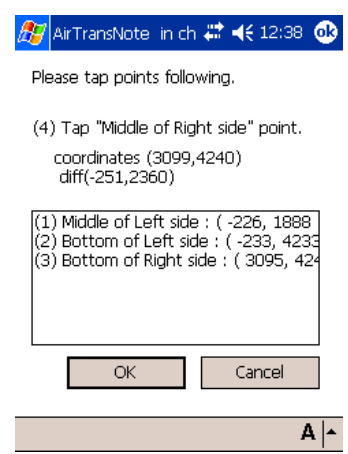

(d)

Figure 5: Snapshots of a transmitter

Inc. The first prototype of the transmitter had a preference window which allows a user to set the server IP address and student ID (Figure 5 (a)), a $\log$ confirmation window (Figure 5 (b)), a note viewer (Figure 5 (c)), and a calibration wizard (Figure 5 (d)). However, we found out that this prototype required the student to tap the touchsensitive screen with a stylus and was thus difficult to use. The revised system was designed to work without a stylus. The software of the transmitter automatically checks the current IP address and connects to an appropriate server.

It is difficult to setup a server IP address and student ID for each transmitter via the preference window. We therefore implemented an administration tool. We added the transmitter to the server port for initial configuration. The administration tool first finds the transmitter, then connects to the port and configures the devices in a remote operation. It also performs version control of the system and re-installment of transmitters. The server IP address and student ID are stored in the system registry key of PocketPC.

\subsubsection{Manager (server)}

The manager is implemented on Java2 with zooming toolkit Piccolo [5]. We designed the interface for both the pen and the mouse, because large displays with a touch sensor panel may become more common in the near future.

In order to show notes in paper sheet images, the teacher needs to prepare the sheet images in advance. An arbitrary image file such as JPEG or PNG can be converted into a "book file" format. The book file also stores some marks for recognition purpose. If the sheet image includes pixels of non-gray colors, the editor generates de- fault marks in the corresponding areas of the pixels. The marks can also be generated manually, with reference to actual notes. After generating and grouping the marks, the teacher sets values for each mark and stores them in the book file. When the teacher opens the book file, the recognition module activates the marks.

\section{Related work}

Much of the previous work on sharing handwritten notes and their applications in e-learning has been based on a device-oriented approach. NotePals 6 . 7] is a PDA-based note-sharing system that captures, and provides access to, handwritten notes and documents of interest to a work group. Notes in the shared repository can be reused by other people. Landay [8] integrated CrossPad ${ }^{1}$ with NotePals but the system requires synchronization to upload notes. In addition, CrossPad is not the best system for daily use in current Japanese classrooms because of its size and weight. SEGODONPDA [9, 10] also uses a PDA as a medium for notesharing. SEGODON-PDA provides both keyboard input and tap-based input for efficient note-taking. Students download class materials using a PDA connected to a wireless LAN, and to share a window of text editor with a cursor for presentation. SEGODON-PDA promotes overall learning including class preparation and review, but it requires a higher level of PDA operating skills than AirTransNote. Tamura and colleagues developed an e-learning system based on TabletPC and tested it in elementary schools [11]. This was done as part of a project initiated by the Center for Educational Computing (CEC) in Japan. The system provides an automatic recognition engine that 


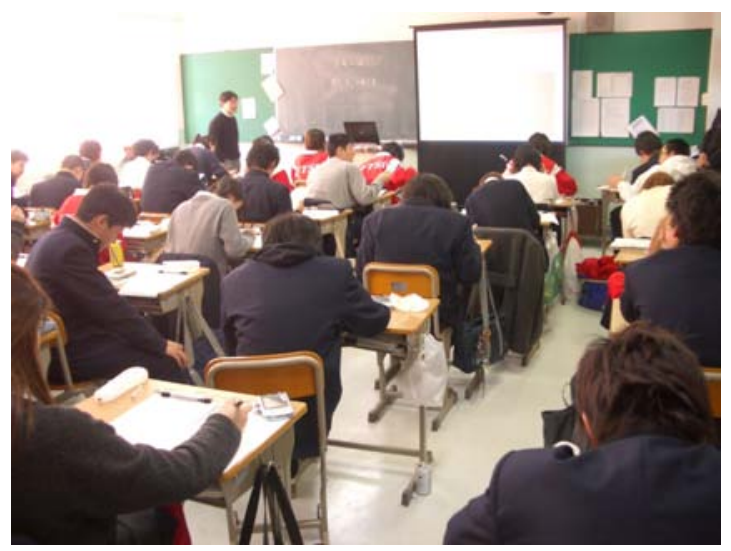

(a)

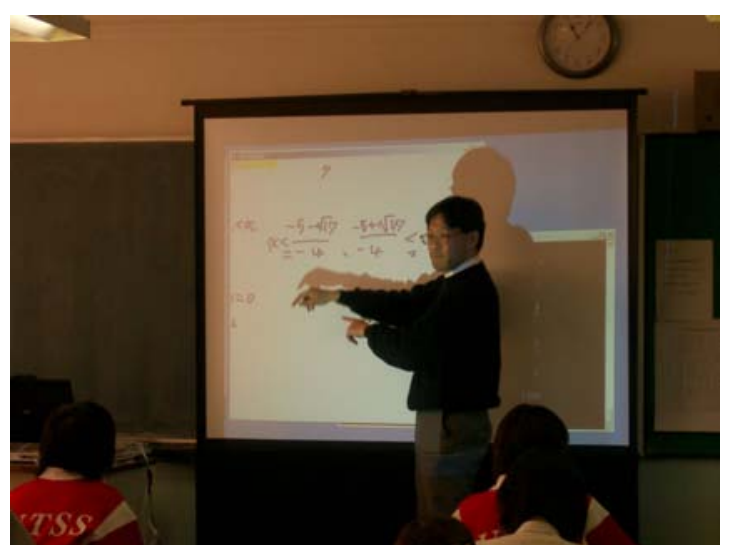

(b)

Figure 6: Feasibility study

recognizes both numbers and Japanese characters. The system enables interactive responses to digital content. But this system also implies a matter of deployment in classroom environment.

The system most similar to ours was described by Maruyama and colleagues [12]. This system was developed by Hitachi Public System Service Co., Ltd., in another CEC project. The system uses Anoto Pen ${ }^{2}$ to record student answers, and the data are synchronized among computer cradles. This system is also based on a paper-centric approach and does not require the use of PDAs to transfer note data. However, it requires the use of paper with a special dotted pattern, and the data are not updated continuously. In addition, the task of connecting many cradles to the teacher's PC can be difficult and time-consuming. Bayon and colleagues [13] also point out that the cost of connecting devices together is high in a real-world school environment. AirTransNote has the following three advantages: (1) it enables real-time updates, (2) it minimizes the set-up time, and (3) it can be scaled to accommodate a different number of clients.

\section{Preliminary feasibility study}

To investigate the effectiveness and practicability of our system, we performed a preliminary feasibility study with 40 subjects. All subjects were first-year high school students (16 males and 24 females). We chose a math class (each class period is 45 minutes). The students first worked on printed questions with digital pens, and then the teacher explained each question.

At the beginning of the class, we distributed digital pens and PDAs, and explained how to use them. The instructions were given in five minutes because of limited class time. We used a 3.2-GHz portable notebook PC as a server and 40 PDAs (Pocket PC 2003, IEEE802.11b wireless LAN-enabled) as transmitters. The server was connected to a local private network by using a 100-base-TX LAN interface.

We asked the students to respond to a questionnaire in the last five minutes of the class. The questionnaires included both multiple-choice and open-ended questions (see Table 1).

\section{Result}

None of the 40 students had any trouble using the system, except for the initial connection set-up. One of the students drew doodles, but he stopped after the teacher erased the doodles on the screen.

We were worried about the bandwidth of the wireless network-whether it was sufficient to cope with note data from 40 transmitters. But the system worked without any problem. It is a benefit of the stroke data size which is relatively small. Figure 6 (a) and (b) show scenes from the class. The teacher showed individual students' answers to the class and explained solutions. However, contrary to our expectation, the teacher seldom used the computer display for browsing. The teacher preferred to look at the students and their answer sheets to specify typical answers for explanation. This means that the browsing interface in our system is still difficult to use in the real world.

${ }^{2}$ http://www.anotofunctionality.com/navigate.asp 
Table 1: Questionnaire items

\begin{tabular}{|lll|}
\hline Q1. & Did you enjoy the system? & Yes $<5,4,3,2,1>$ No \\
\hline Q2. & Did you feel stress while using the system? & Yes $<5,4,3,2,1>$ No \\
\hline Q3. & Did you feel uncomfortable using the system? & Yes $<5,4,3,2,1>$ No \\
\hline Q4. & Do you want to use the system again? & Yes $<5,4,3,2,1>$ No \\
\hline Q5. & $\begin{array}{l}\text { Do you think that replaying notes using a time-line bar } \\
\text { can be helpful to you and the teacher? }\end{array}$ & Yes $<5,4,3,2,1>$ No \\
\hline Q6. & Do you think that this system improves the quality of learning? & Yes $<5,4,3,2,1>$ No \\
\hline
\end{tabular}

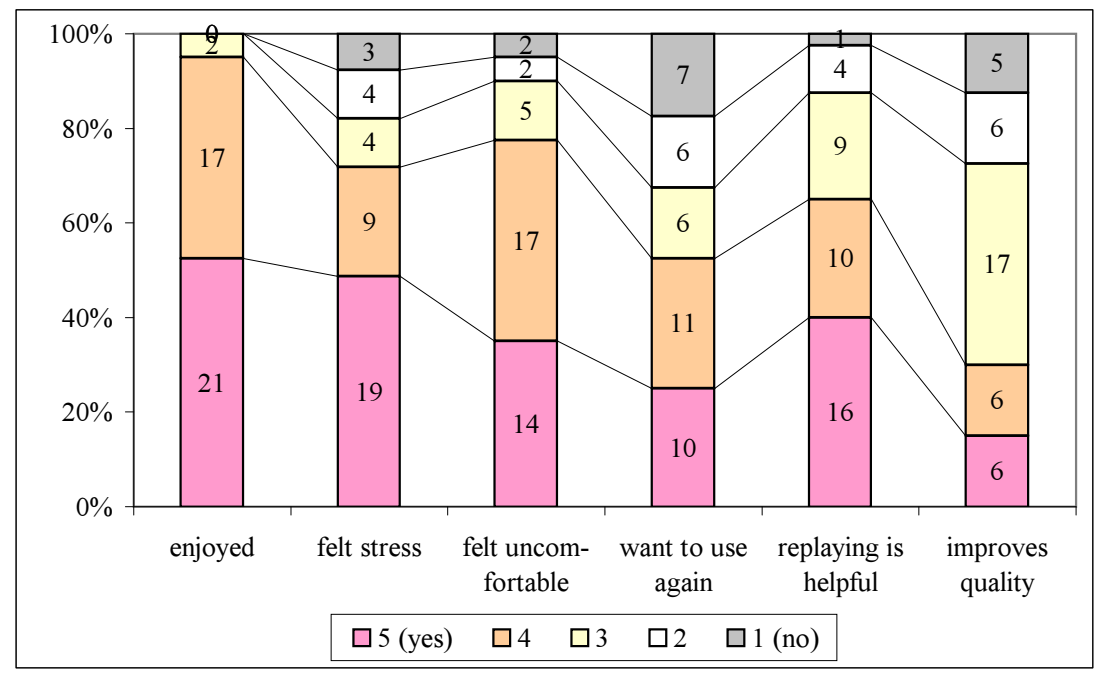

Figure 7: Questionnaire results

Although improving the browser interface is one solution, we should also look for ways of focusing on to the student panel in a natural way.

The questionnaires (see Figure 7) showed that most of the students were interested in the system and enjoyed the session. Some students were surprised by the system and digital pen devices. However, more than half of the students in the class said they were not comfortable with sharing their notes, particularly when they did not know the answer. This feeling of uneasiness was emphasized by the properties of the pens we used. Because the pens were ball-point pens, the students could not erase their answers. We believe that the system is useful but the difference of the pen is quite important to students.

To improve the system, we need to consider the following issues.

- How to ensure consistency between notes on paper and digital data and at the same time allow students to erase their notes.

- How to present to students the teacher's feedback in the form of advice, annotations, and comments.
To provide feedback to students, the teacher can use the display of the PDA, and this function is implemented in our system (see Figure 5 (c); an annotation is shown in red), but this solution is not perfect because it does not free the student from the need to use electronic devices and it is not suitable for a paper-centric approach. In addition to this, the teacher does not have enough time to manually reply to all answers during the class. The feedback should also be presented in the same place of the paper.

\section{Conclusion}

We developed a paper-centric collaborative learning system called AirTransNote. AirTransNote is designed to augment the traditional classroom instruction and enhance student-teacher interaction through note-sharing. Using this system, teachers can closely monitor their students' process. The system has a recognition module that helps the teacher summarize class progress as well as activities of each student. The system is also useful to students because it improves the quality of 
the learning process by making it possible for students to share ideas, solutions, and activities. Our preliminary study indicates that this approach to learning in the classroom is feasible. We believe that the paper-centric approach is appropriate because the pen minimizes the amount of cognitive effort required by the use of specific devices and maximizes collaboration through note-sharing. In the future, we want to improve the recognition module to better support teachers.

\section{Acknowledgment}

We would like to thank Hiroyuki Sakamoto for his comments and arrangements for our experiments. This work was supported in part by a grant-in-aid for Scientific Research (15020216, 14780187).

\section{References}

[1] http://www.manabinet.jp/it_ed.pdf (in Japanese).

[2] Jun Rekimoto, Yuji Ayatsuka, Hitoraka Uoi, and Toshifumi Arai. Adding Another Communication Channel to Reality: An Experience with a ChatAugmented Conference. In CHI 98 conference summary on Human factors in computing systems, pages 271-272, April 1998.

[3] Seiko Instruments USA Inc. InkLink. http://www.siibusinessproducts. com/products/link-ir-p.html.

[4] François Guimbretière and Terry Winograd. FlowMenu: Combining Command, Text, and Data Entry. In Proceedings of ACM User Interface Software and Technology 2000 (UIST 2000), pages 213-216, November 2000.

[5] Benjamin B. Bederson, Jesse Grosjean, and Jon Meyer. Toolkit Design for Interactive Structured Graphics. Technical Report HCIL-2003-01, CSTR-4432, UMIACS-TR-2003-03, Institute for Advanced Computer Studies, Computer Science Department, University of Maryland, January 2003.

[6] Richard C. Davis, James A. Landay, Victor Chen, Jonathan Huang, Rebecca B. Lee, Francis Li,
James Lin, Charles B. Morrey III, Ben Schleimer, Morgan N. Price, and Bill N. Schilit. NotePals: Lightweight Note Sharing by the Group, for the Group. In Proceedings of the SIGCHI conference on Human factors in computing systems, pages 338-345, May 1999.

[7] Richard C. Davis, James Lin, Jason A. Brotherton, James A. Landay, Morgan N. Price, and Bill N. Schilit. A framework for sharing handwritten notes. In Proceedings of the 11th annual ACM symposium on User interface software and technology (UIST98), pages 119-120, November 1998.

[8] James A. Landay. Using Note-Taking Appliances for Student to Student Collaboration. In Proceedings of the 29th ASEE/IEEE Frontiers in Education Conference, pages 12c4-15-20, November 1999.

[9] Takashi Yoshino and Jun Munemori. SEGODON: Learning Support System that can be Applied to Various Forms. In Claude Ghaoui, editor, EEducation Applications: Human Factors and Innovative Approaches, pages 132-152. Information Science Publishing, February 2004.

[10] Tomohiro Shigenobu, Takahiro Noda, Takashi Yoshino, and Jun Munemori. SEGODON-PDA: Flexible Lecture Support System Using Wireless LAN and PDAs. IPSJ Journal, 45(1):255-266, January 2004. (in Japanese).

[11] Hiroaki Tamura, Naomi Iwayama, Hiroshi Tanaka, Katsuhiko Akiyama, and Kazushi Ishigaki. An Implementatin of Handwriting Learning Materials on Tablet-PC and its Practical Studies in an Elementary School. In Proceedings of Interaction 2004, pages 47-52. IPSJ Symposium Series Vol. 2004, No. 5, March 2004. (in Japanese).

[12] http://www.cec.or.jp/e2a/other/ 04PDF/b1.pdf (in Japanese; the URL of the project page is http://www.hitachi-ks. co.jp/cec/index.html).

[13] Victor Bayon, Tom Rodden, Chris Greenhalgh, and Steve Benford. Going Back to School: Putting a Pervasive Environment into the Real World. In 1st International Conference on Pervasive Computing (LNCS 2414), pages 69-83, August 2002. 